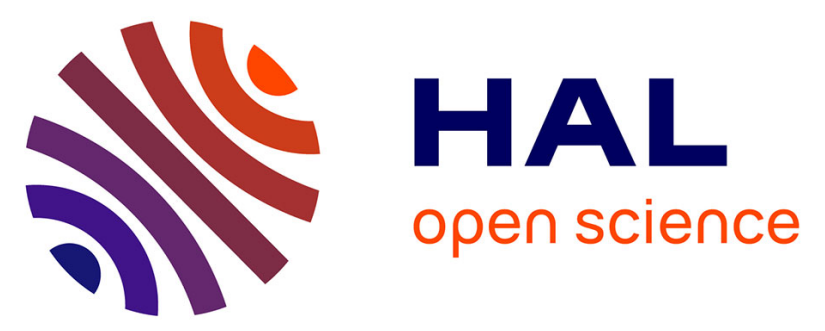

\title{
MRI Texture Analysis for Differentiation Between Healthy and Golden Retriever Muscular Dystrophy Dogs at Different Phases of Disease Evolution
}

Dorota Duda, Marek Kretowski, Noura Azzabou, Jacques De Certaines

\section{To cite this version:}

Dorota Duda, Marek Kretowski, Noura Azzabou, Jacques De Certaines. MRI Texture Analysis for Differentiation Between Healthy and Golden Retriever Muscular Dystrophy Dogs at Different Phases of Disease Evolution. 14th Computer Information Systems and Industrial Management (CISIM), Sep 2015, Warsaw, Poland. pp.255-266, 10.1007/978-3-319-24369-6_21 . hal-01444470

HAL Id: hal-01444470

https://hal.inria.fr/hal-01444470

Submitted on 24 Jan 2017

HAL is a multi-disciplinary open access archive for the deposit and dissemination of scientific research documents, whether they are published or not. The documents may come from teaching and research institutions in France or abroad, or from public or private research centers.
L'archive ouverte pluridisciplinaire HAL, est destinée au dépôt et à la diffusion de documents scientifiques de niveau recherche, publiés ou non, émanant des établissements d'enseignement et de recherche français ou étrangers, des laboratoires publics ou privés.

\section{(c)(1)}

Distributed under a Creative Commons Attribution| 4.0 International License 


\title{
MRI texture analysis for differentiation between healthy and Golden Retriever Muscular Dystrophy dogs at different phases of disease evolution
}

\author{
Dorota Duda ${ }^{1}$, Marek Kretowski ${ }^{1}$, Noura Azzabou ${ }^{2,3}$, and Jacques D. de \\ Certaines $^{2,3}$ \\ ${ }^{1}$ Faculty of Computer Science, Bialystok University of Technology \\ Wiejska 45a, 15-351 Bialystok, Poland \\ ${ }^{2}$ Institute of Myology, Nuclear Magnetic Resonance Laboratory, Paris, France \\ ${ }^{3}$ CEA, I2BM, MIRCen, NMR Laboratory, Paris, France \\ \{d.duda, m.kretowski\}@pb.edu.pl
}

\begin{abstract}
In this study, a texture analysis is applied to T2-weighted Magnetic Resonance Images (MRI) of canine pelvic limbs in order to differentiate between Golden Retriever Muscular Dystrophy (GRMD) dogs and healthy ones. The differentiation is performed at three phases of canine growth and/or disease development: 2-4 months (the first phase), 5-6 months (the second phase), and 7 months and more (the third phase). Eight feature extraction methods (statistical, model-based, and filterbased) and five classifiers are tested. Four types of muscles are analyzed: the Extensor Digitorum Longus (EDL), the Gastrocnemius Lateralis (GasLat), the Gastrocnemius Medialis (GasMed) and the Tibial Cranialis (TC). The experiments were performed on five healthy and five GRMD dogs. Each of the muscles was considered separately. The best classification results were $95.81 \%$ (the EDL muscle), $97.19 \%$ (GasLat), and $91.37 \%$ (EDL) correctly recognized cases, for the first, second and third phase, respectively. These results were obtained with an SVM classifier.
\end{abstract}

Keywords: Golden Retriever Muscular Dystrophy (GRMD), Duchenne Muscular Dystrophy (DMD), texture analysis, tissue characterization, muscles, classification, dog, MRI T2, Computer-Aided Diagnosis (CAD)

\section{Introduction}

The Golden Retriever Muscular Dystrophy (GRMD) canine model is acknowledged to be the closest to human Duchenne Muscular Dystrophy (DMD, the most common and most severe form of muscular dystrophy) [1]. For this reason it is commonly used for various pre-clinical and therapeutic trials. In research on GRMD development or its response to treatment, great hope is placed in Magnetic Resonance (MR) image analysis, performed with various (semi)automatic, 
computer-aided techniques. Other diagnostic methods (such as needle biopsy followed by histopathological analysis), while providing more reliable diagnoses, are to be avoided here. They can alter muscle integrity and weaken muscles which are already degenerated and poorly functioning due to disease progression.

It has been shown that image texture can be a very important source of information contained in the image [2]. Texture analysis has already been successfully applied to many diagnostic problems, concerning a variety of tissue types and/or imaging modalities. A broad review of texture analysis methods and applications in the field of medical image classification can be found in several articles [3-5].

The aim of this study is to assess the potential of various MRI texture analysis techniques (statistical, model-based, and filter-based) for characterization of different types of muscles in canine pelvic limbs: the Extensor Digitorum Longus (EDL), the Gastrocnemius Lateralis (GasLat), the Gastrocnemius Medialis (GasMed) and the Tibial Cranialis (TC). In total, eight methods of texture analysis are used. A texture-based differentiation between healthy and GRMD dogs is performed at three different phases of canine growth and/or disease development: 2-4 months (the first phase), 5-6 months (the second phase), and 7 months and more (the third phase). The division into three phases is made in reference to histological changes in muscle structure resulting from disease progression. As early as the first 3-4 months of age, GRMD skeletal muscles display hypercontracted, degenerating or regenerative isolated fibers [6]. After this period (up to the age of about 6 months), the number of degenerating necrotic fibers dramatically increases [7]. At this stage, varying degrees of fibrosis and adipose infiltration can occur. Finally, from 6 months of age, GRMD is in its third phase, which is characterized by rather slow muscular changes, with a lower level of degeneration and regeneration, and progressive intensity of fibrosis and fat tissue infiltration [8]. As such changes may alter the structure of each muscle type in different ways, each type of muscle is considered separately in this study.

The experiments are repeated using five well-known classifiers: Support Vector Machines, Back-Propagation Neural Networks, Logistic Regression, Random Forest, and Adaptive Boosting. To the best of our knowledge, this is the first comparative study of texture-based classification of the Canine Hindlimb Muscles involving such a large number of texture analysis techniques and classifiers, and comparing the usefulness of the textural information extracted from different types of muscles and at each phase of canine growth / disease development.

\section{Related Work}

Texture analysis of different human muscle types has already been studied in some works (for a review, please refer to $[9,10]$ ). However, there are few studies dealing with its application to GRMD tissue characterization or characterization of disease development over time.

One of the earliest studies on MRI texture analysis applied to characterization of dystrophic muscles was presented in [11]. The work assessed the potential of several texture analysis techniques (based on the gray-level histogram, 
co-occurrence matrices, gradients, and run length matrices) in comparison to the potential of visual analyses of the same ROIs carried out by several radiologists (each radiologist analyzed a single ROI). Textural features were extracted from T1-weighted images and analyzed by Correspondence Factorial Analysis (CFA). Automated classification of dystrophic and healthy patients resulted in $70 \%$ and $86 \%$ sensitivity and specificity, respectively. The results achieved by the radiologists assessments were less accurate (sensitivity of $56 \%$ and specificity of $71 \%)$.

The aim of another work [12] was to evaluate the usefulness of MRI texture analysis methods in the characterization of diseased and healthy calf muscles. In addition, these methods were compared to standard radiological evaluation. The authors tested a total of 282 textural features (statistical and model-based) derived from T1-weighted images. Four different classes of calf muscle were considered, depending on the severity of the pathology affecting the muscles. The classification results obtained with the best (selected) 7 textural features were in $80 \%$ agreement with the categorization made by the radiologists.

Fan et al. [13] analyzed different MRI biomarkers (non-textural MR imagingbased and texture analysis-based) for seven muscles of the proximal pelvic limbs in GRMD and healthy control dogs. All the dogs were imaged at 3, 6, and 912 months of age. The aim of the study was to quantify longitudinal disease progression and to differentiate between two groups of dogs in different stages of canine growth and/or disease development. Texture features, based on the gray-level histogram and run length matrices, were calculated from T2-weighted images. The statistical test (Mann-Whitney-Wilcoxon) found all the texture features to be significantly higher in the GRMD dogs than in the healthy controls, in each dog age group. Moreover, classification based on Linear Discriminant Analysis (LDA) showed that RLM-based texture features had on average better discriminatory power than other MRI biomarkers (such as fat content and T2 relaxation time). Nevertheless, for certain muscles, texture features did not show significant correlations with the histopathology indices.

In the work [14] texture analysis of T2-weighted MR images was used to describe the structure of thigh soft tissues in five groups of athletes (representing differently loading sport types: repetitive non-impact, repetitive low impact, odd impact, high impact, and high magnitude) and non-athletes. Five thigh muscles at two anatomical levels of the dominant leg were characterized with co-occurrence matrix-based texture features. Statistical analyses showed differences in thigh muscle textures, especially between the athletes performing high impact and odd impact loading sports.

Another study [15], performed on a similar database (five athlete groups and non-athletic controls), analyzed the differences in MRI texture properties of hip muscles (on T1-weighted images). Four different muscle types were considered in the study. Six methods for extraction of texture features were used to characterize muscular tissues (based on the gray level histogram, gradient matrices, co-occurrence matrices, run length matrices, wavelets, and an autoregressive model). Significant differences in texture properties between athletes 
and non-athletes were observed for the four considered sport types for at least one muscle type. Only between the non-magnitude loading athletes and the nonathlete controls no significant differences were found in muscle texture.

\section{Texture-Based Classification of the Canine Hindlimb Muscles}

Two stages can be distinguished in a typical system for texture-based classification of medical images [16]. The first stage is preparation of the learning set used to construct the classifiers. In this stage a database of images, as large as possible, is gathered and preprocessed (if necessary). Then the Regions of Interest (ROIs, the image regions subjected to the analysis) are delineated, either manually, or by (semi)automated segmentation methods. Each ROI is assigned a label that refers to a tissue class (e.g., healthy or pathologically changed) and is based on a verified diagnosis. Afterwards various methods are applied in order to characterize each ROI. In our case, these methods are based on texture analysis and result in the creation of vectors of textural features. Each feature is a numerical measure of a specific texture property, such as coarseness, granularity, regularity, entropy, or frequency of stripes, waves, edges, etc. Labeled feature vectors form a training set, based on which several classifiers can be constructed.

Once the classifiers are created, the system can be used for recognition of new cases that have not yet been diagnosed. In this second stage of operation of the system, a suspicious image region is delineated by a physician or segmented (semi)automatically by the methods incorporated in the system. Then it is characterized by texture features, the same as were used in the first stage. Finally, the system classifiers are used to indicate the most probable tissue class. These two stages of work with the system are depicted schematically in Figure 1. This work-flow was used in our experiments, aimed at recognition of healthy and GRMD dogs based on muscle MRI texture analysis.

\subsection{Database Description}

The experiments were performed on the database provided by [17]. Five GRMD dogs and five healthy controls were considered in the study. The dogs were bred in a dedicated gene therapy facility at the National Veterinary School of Alfort, France. All procedures were carried out in accordance with the Guide for the Care and the Use of Laboratory Animals and approved by the Institutional Animal Use and Care Committee, in accordance with European legislation regarding the use of laboratory animals. The image database was created at the Nuclear Magnetic Resonance Laboratory of the Institute of Myology, Pitie-Salpetriere University Hospital in Paris, France (its full description, as well as a description of all the acquisition protocols, can be found in [17]). Each acquisition was performed on a 3T Siemens Magnetom Trio TIM imager/spectrometer (Siemens Healthcare, Erlangen, Germany) with a standard circularly polarized extremity coil. The in-plane resolution was $0.56 \mathrm{~mm} \times 0.56 \mathrm{~mm}$, the slice thickness 


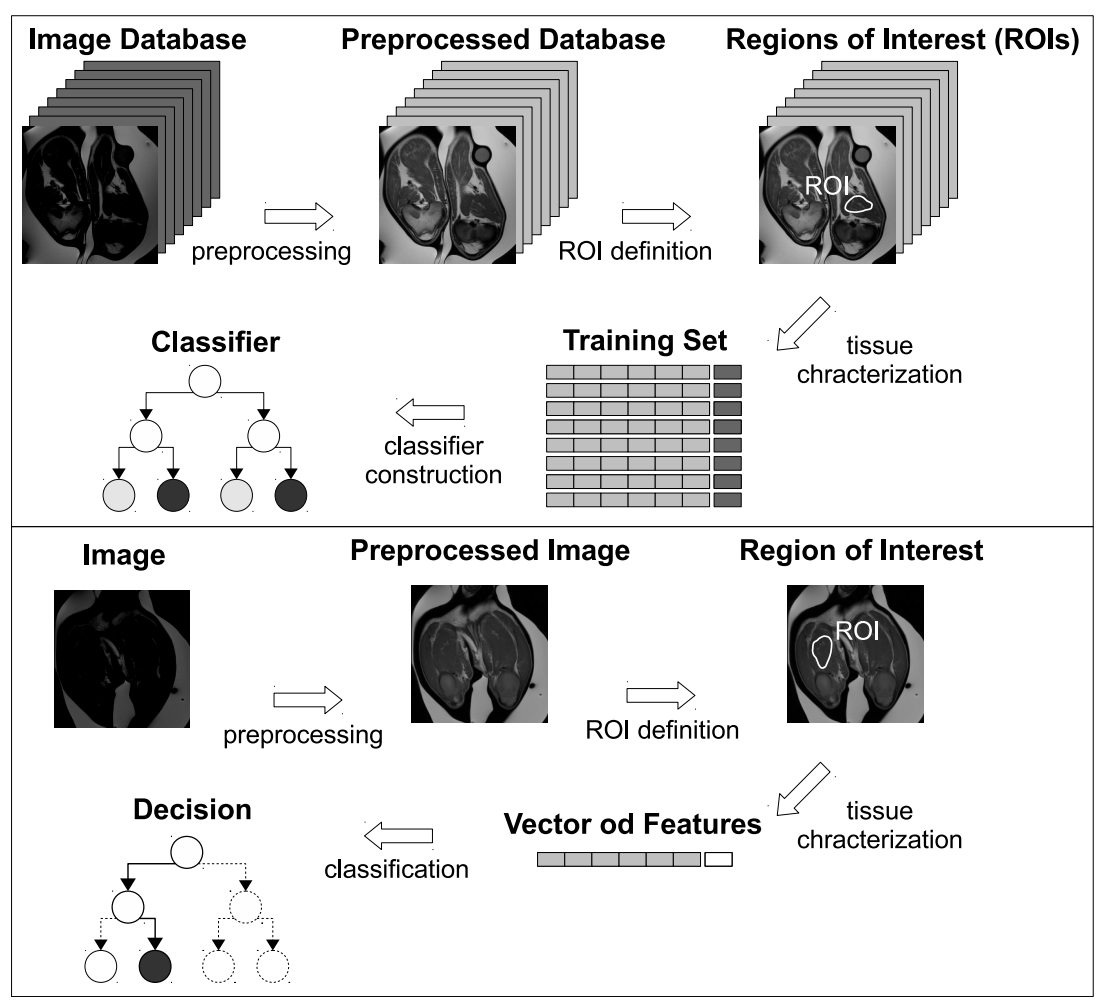

Fig. 1. Typical system for texture-based classification. Two stages of operation of the system: training (the upper part of the figure) and learning (the lower part)

was $3 \mathrm{~mm}$, and the inter-slice gap was $7.5 \mathrm{~mm}$. The slice orientation was axial with respect to the long axis of the muscle. Anesthesia was induced in the dogs with propofol (Rapinovet ${ }^{\circledR}$, Schering-Plough), injected intravenously at a dose of $0.65 \mathrm{ml} / \mathrm{kg}$. The T2-weighted Spin Echo sequences (used in our analyses) were acquired with the following parameters: repetition time $\mathrm{TR}=3,000 \mathrm{~ms}$, echo time TE1 $=6.3 \mathrm{~ms}$, and TE2 $=50 \mathrm{~ms}$. Each image series comprised from 12 to 14 images. For each dog, from 3 to 5 examinations were performed over a maximum of 14 months. In total, 38 examinations were used in the study. Each examination was assigned to one of the three considered phases of canine growth and/or disease development. In total, 14, 9, and 15 examinations were available for the first, second and third phase, respectively.

Only 4 types of muscle were considered in our analyses (EDL, GasLat, GasMed, and TC). Other muscle types were ignored because they occupied very small areas on the images (less than 50 pixels within a ROI), or due to unsuitable geometry (areas that were too narrow). For each muscle a minimum of 3 images were segmented. Only ROIs larger than 40 pixels and having each 
Table 1. Average sizes of ROIs used in our experiments

\begin{tabular}{l}
\hline muscle \class \\
\hline EDL \\
GasLat \\
GasMed \\
TC \\
\hline
\end{tabular}

\begin{tabular}{ll}
\hline \multicolumn{2}{c}{ Phase 1 } \\
\hline GRMD & healthy \\
\hline 102 & 127 \\
144 & 123 \\
244 & 267 \\
116 & 162 \\
\hline
\end{tabular}

\begin{tabular}{ll}
\hline \multicolumn{2}{c}{ Phase 2} \\
\hline GRMD & healthy \\
\hline 156 & 213 \\
204 & 170 \\
266 & 395 \\
241 & 248 \\
\hline
\end{tabular}

\begin{tabular}{ll}
\hline \multicolumn{2}{c}{ Phase3 } \\
\hline GRMD & healthy \\
\hline 142 & 271 \\
158 & 212 \\
311 & 420 \\
227 & 314 \\
\hline
\end{tabular}

Table 2. Numbers of ROIs used in our experiments

\begin{tabular}{|c|c|c|c|c|c|c|}
\hline \multirow[b]{2}{*}{ muscle \class } & \multicolumn{2}{|c|}{ Phase 1} & \multicolumn{2}{|c|}{ Phase 2} & \multicolumn{2}{|c|}{ Phase3 } \\
\hline & GRMD & healthy & GRMD & healthy & GRMD & healthy \\
\hline EDL & 96 & 104 & 77 & 47 & 95 & 149 \\
\hline GasLat & 67 & 46 & 38 & 28 & 45 & 90 \\
\hline GasMed & 82 & 68 & 45 & 37 & 64 & 115 \\
\hline $\mathrm{TC}$ & 104 & 107 & 92 & 67 & 105 & 178 \\
\hline
\end{tabular}

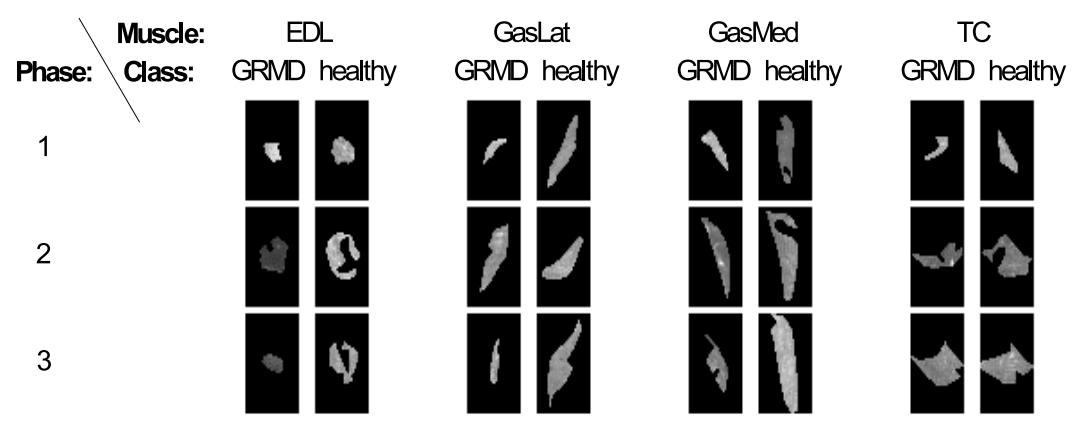

Fig. 2. Examples of ROIs for each tissue type, tissue class, and phase of canine growth and/or disease development. The size of each black square is $30 \times 47$ pixels

dimension greater than four pixels were kept for further analysis. The numbers of analyzed ROIs and their average dimensions are given in Tables 1 and 2, respectively. In Figure 2 exemplary ROIs for each muscle type and for each muscle class (GRMD, healthy) are presented.

Due to the differences in average image intensities between different examinations, and even between different images within the same series, the images had to be preprocessed. The preprocessing consisted in a linear image transformation, performed separately for each image, so that the reference object was of the same average pixel intensity on all images. Owing to this procedure the gray level interval covering all the pixels belonging to the ROIs did not exceed 256 values. 


\section{$3.2 \quad$ Texture Analysis}

Eight texture analysis methods (statistical, model-based and filter-based) were considered for our investigation. They were implemented in our homemade software MIP (Medical Image Processing). In total, 39 features were calculated:

- average, variance, skewness, and kurtosis - first order statistics (FOS), obtained from a gray-level histogram,

- normalized autocorrelation coefficient (AC) [18],

- average, variance, skewness, kurtosis, from the gradient matrix (GM) [19],

- energy, inverse difference moment, entropy, correlation, sum average, difference average, sum variance, difference variance, sum entropy, difference entropy, and contrast, from the co-occurrence matrices (COM) [20],

- short run emphasis, long run emphasis, gray level non-uniformity, run length non-uniformity, fraction of image in runs, low gray level runs emphasis, high gray level runs emphasis, and run length entropy, from the run length matrices (RLM) [21-23],

- average, entropy, angular second moment, inverse difference moment, and contrast, form the gray level difference matrices (GLDM) [24],

- fractal dimension based on the fractional Brownian motion model (FB) [25],

- entropy of an image region filtered with the following pairs of the Laws' masks: (E3L3 and L3E3), (S3L3 and L3S3), (S3E3 and E3S3), (E3E3 and E3E3), and (S3S3 and S3S3) [26].

Each of the aforementioned sets of features was considered separately. In addition, a set of all the possible features (calculated with the eight enumerated texture analysis methods) was tested. The use of a relatively large number of texture characteristics was dictated by two facts. Firstly, various stages of the disease were considered, and each stage results in other changes in the muscle structure. These latter alter in different way the muscle texture of an MRI image. Secondly, the observed changes are not the same for each type of muscle. In turn, each texture feature is a numerical measure of other texture property, related to the type of muscle and and/or disease development. A detailed comparative study on various MRI texture analysis techniques in muscular dystrophy of GRMD dogs, explaining the histological meaning of MRI textures, and justifying the use of various texture features can be found in [9]).

The choice of parameter settings for each method (such as maximum pixel distance) was strictly dependent on the properties of the ROIs. Most of the ROIs were rather thin and narrow, so the use of some methods would have required further elimination of dozens of ROIs. For example, in the first phase of canine growth, the percentages of ROIs unsuitable for Laws' method with a mask of size $5 \times 5$ were $31.50 \%, 42.48 \%, 18.67 \%$, and $28.91 \%$ for EDL, GasLat, GasMed, and TC, respectively. A similar problem pertained to the co-occurrence matrix-based, gray level matrix-based, autocorrelation-based, and fractal-based approach. For all of them the maximum considered pixel distance was set at 2 .

The co-occurrence matrices and the gray level difference matrices were constructed separately for 4 standard directions $\left(0^{\circ}, 45^{\circ}, 90^{\circ}\right.$, and $\left.135^{\circ}\right)$ and for 
2 considered distances between pixel pairs ( 1 and 2). The same four directions and/or two pixel distances were also considered in the run length matrix-based method and during calculation of the normalized autocorrelation coefficients. For all four aforementioned methods, features corresponding to different directions of pixel arrangement and/or different pixel distances were averaged. All possibilities of averaging (or not) of the features corresponding to different directions and/or to different pixel distances were tested.

\subsection{Classification}

Classification experiments were performed with Weka software [27]. Five classifiers were used to compare the potential of different feature sets and the usefulness of the information extracted from each of the 4 considered muscles:

- Support Vector Machines (SVM) [28] using a Sequential Minimal Optimization (SMO) algorithm [29] and a second degree polynomial kernel,

- Neural Network (NN) [30] with a backpropagation and sigmoidal activating function, having a hidden layer wherein the number of neurons was equal to the average value of the number of features and the number of classes,

- Logistic Regression (LR) [31],

- Random Forest (RF) [32] comprising a set of 50 trees,

- Adaptive Boosting (AB) [33] using the C4.5 tree [34].

Classification accuracies were estimated by 10 -fold cross-validation, repeated 10 times. The 100 partial results were averaged.

\section{Results and Discussion}

The best results obtained by each classifier for each type of muscle and at each phase of canine growth / disease progression are presented in Table 3.

It can be seen from Table 3 that the usefulness of textural information is different for each muscle. It also changes over time, with canine growth (and/or disease development). In the earliest phase of canine life (2-4 months), the most important information could be extracted from the EDL muscle texture. For all the classifiers, the best classification result at this first phase was obtained for this muscle - from $93.44 \%$ for the AB classifier to $95.81 \%$ for the SVM. For comparison, at the same phase, only $81.70 \%$ (AB) to $83.91 \%$ (SVM) of cases were correctly recognized with the textural features extracted from the GasLat muscle. In contrast, the texture of the GasLat muscle proved the most useful (or nearly the most useful) for classification at the second phase of canine life. The highest percentage of correctly diagnosed cases (97.19\%) was observed for the GasLat-based textural features combined with the SVM classifier. Still, at the second phase, it was the GasMed muscle that outperformed the other muscles in terms of classification when the $\mathrm{RF}$ and the $\mathrm{AB}$ classifier were used. In the latter case, the GasLat muscle gave slightly inferior results (by about 1\%-2\%) than the GasLat muscle. Finally, at the third phase, no clear advantage of any 
Table 3. Differentiation between healthy and GRMD dogs at different phases of canine growth / disease evolution. The best classification accuracies [\%] (and standard deviations) achieved with five different classifiers ("Cl") and for each considered muscle of canine pelvic limbs. Each result is followed by the corresponding texture analysis method

\begin{tabular}{llllll}
\hline \multirow{2}{*}{ Cl } & \multirow{2}{*}{ Phase } & \multicolumn{5}{c}{ Muscle } \\
\cline { 2 - 6 } & & EDL & GasLat & GasMed & TC \\
\hline \multirow{2}{*}{ SVM } & 1 & $95.81(4.63)$ RLM & $83.91(11.44)$ GLDM & $86.72(8.89)$ All & $85.40(8.42)$ All \\
& 2 & $90.72(8.10)$ COM & $97.19(6.38)$ COM & $94.92(7.91)$ COM & $91.65(7.95)$ COM \\
& 3 & $91.37(4.79)$ RLM & $89.45(9.00)$ COM & $89.55(6.82)$ RLM & $90.81(5.78)$ All \\
\hline \multirow{2}{*}{ NN } & 1 & $94.83(5.22)$ RLM & $82.87(12.01)$ GLDM & $83.84(9.61)$ All & $85.69(7.75)$ All \\
& 2 & $91.62(7.52)$ COM & $96.93(6.20)$ COM & $93.33(8.86)$ All & $89.10(7.32)$ All \\
& 3 & $90.81(5.87)$ COM & $87.82(8.38)$ All & $89.81(7.12)$ RLM & $89.83(5.86)$ COM \\
\hline \multirow{2}{*}{ LR } & 1 & $94.72(5.78)$ RLM & $83.36(11.34)$ GLDM & $83.79(9.64)$ All & $83.19(6.67)$ COM \\
& 2 & $89.83(9.66)$ All & $95.50(8.11)$ COM & $91.38(9.40)$ FOS & $87.30(9.93)$ FOS \\
& 3 & $86.82(6.38)$ RLM & $89.76(7.34)$ RLM & $86.83(7.45)$ COM & $89.58(5.13)$ COM \\
\hline \multirow{2}{*}{ RF } & 1 & $93.59(6.22)$ All & $83.15(10.26)$ COM & $81.84(9.02)$ RLM & $83.51(7.86)$ COM \\
& 2 & $90.83(8.58)$ All & $91.36(11.15)$ RLM & $93.14(8.63)$ RLM & $89.05(8.80)$ RLM \\
& 3 & $88.94(6.16)$ COM & $88.03(7.62)$ RLM & $89.24(5.66)$ RLM & $89.82(5.17)$ All \\
\hline \multirow{2}{*}{ AB } & 1 & $93.44(6.52)$ All & $81.70(11.46)$ COM & $84.27(9.38)$ All & $84.65(7.28)$ COM \\
& 2 & $92.08(7.34)$ All & $91.90(10.65)$ COM & $92.83(9.84)$ COM & $86.75(8.36)$ All \\
& 3 & $88.60(6.51)$ COM & $87.82(8.15)$ All & $86.25(8.12)$ RLM & $90.91(5.03)$ COM \\
\hline
\end{tabular}

one muscle could be observed, and the differences in the results obtained with different muscles were the smallest. For the SVM and the NN classifiers, the best results were achieved with the GasLat muscle (91.37\% and $90.81 \%$, respectively). For the $\mathrm{RF}$ and $\mathrm{AB}$ classifiers, the best third-phase results were obtained for the TC muscle ( $89.82 \%$ and $90.91 \%$, respectively).

Given the above results, it can be concluded that the classification results are not strongly correlated with the size of the characterized ROIs. The crucial textural information, in terms of tissue differentiation, could be extracted from even very small ROIs. For example, the EDL muscle occupied the smallest image regions in almost all cases, and it was with this muscle that the classification experiments often gave the best results.

The best overall classification results at the first, second, and third phase were $95.81 \%, 97.19 \%$, and $91.37 \%$ correctly recognized cases, respectively. The significant difference between the two latter phases is not surprising, if we consider that the set of ROIs corresponding to the third phase was two times greater than the one used for the second phase (see Table 1). Direct comparisons between phases could be more reliable if equally numerous datasets were used for each phase-problem. In our case, the original number of ROIs was small, so we preferred not to truncate the datasets.

Finally, it could be seen that the best texture analysis methods were those based on the co-occurrence matrices and on the run length matrices. It is difficult 
to say which of these is better, as their application very often resulted in similar classification accuracies. The use of the COM method led to better results for the GasLat muscle type, whereas the RLM method was the most frequently selected for the TC muscle. In the case of the first- and second-phase classification problem, the sets composed of all the possible textural features (derived from the 8 classification methods) were also frequently selected as the best ones.

\section{Conclusion}

The study investigated the possibility of using MRI texture analysis in the process of distinguishing between healthy and GRMD dogs at various stages of canine growth and/or disease development. Four types of muscle located in canine pelvic limbs were considered: EDL, GasLat, GasMed, and TC. Eight texture analysis methods were used for their characterization, including statistical, model-based, and filter-based methods. The experiments, while conducted on a small sample, showed that several texture analysis methods have great potential. The best ones proved to be those based on co-occurrence matrices and run-length matrices. Satisfactory results were also obtained with the sets comprising features provided by all of the eight considered texture analysis methods. In this case, the calculations required more memory to store data and more time for classifier construction.

The classification results for each muscle were compared in order to evaluate which type of muscle may provide the most useful information in terms of disease detection in different phases of canine growth. At the earliest phase (2-4 months of canine life), the best differentiation between healthy and GRMD dogs was observed for the EDL muscle (for all classifiers used). In the second phase (5-6 months), the GasLat and the GasMed muscles were found to provide slightly better texture characteristics than the other two muscles. Finally, in the third phase ( 7 months and more), the best results corresponded either to the EDL muscle texture analysis (with the SVM and NN classifiers), the TC muscle (RF, $\mathrm{AB}$ ), or the GasLat muscle (LR).

\section{Future Work}

Some problems occurring during our investigations are still unresolved an will be the subject of our future research. For example, the choice of minimum acceptable ROI size remains problematic. Moreover, it is not only the ROI size, but also its shape that determines its suitability for texture analysis. The choice of distances between pixel pairs should also be assessed. Certain texture properties will not be captured when only small distances (such as 1 or 2) are considered for the COM, GLDM, FB, or AC method. If distances were too large (5 or more pixels), many more ROIs would have to be discarded due to their size - too small to guarantee a large enough number of pixel pairs for feature calculation. Having only 10 dogs for the analyses, we had to limit ourselves to consideration of only small distances, in order not to reject too many ROIs. Similar experiments 
should be conducted on a larger data base. It will also be interesting to explore other types of MR images (e.g. T1-weighted, fat-suppressed, acquired using the Dixon technique, diffusion tensor sequences, etc.) and perform a multiparametric texture classification. Other texture analysis methods (such as those based on wavelets, the Fourier transform, or the Gabor transform) could also be tested. Finally, it will be interesting to study the evolution of textural features during canine growth / disease development.

Acknowledgments. This work was performed under the auspices of the European COST Action BM1304, MYO-MRI. It was also supported by grant S/WI/2/2013 from the Bialystok University of Technology, Bialystok, Poland.

\section{References}

1. Kornegay, J.N., Bogan, J.R., Bogan, D.J., Childers, M.K., Li, J., et al.: Canine models of Duchenne muscular dystrophy and their use in therapeutic strategies. Mamm. Genome 23(1-2), 85-108 (2012)

2. Haralick, R. M.: Statistical and structural approaches to texture. Proc. of the IEEE 67(5), 786-804 (1979)

3. Castellano, G., Bonilha, L., Li, L.M., Cendes, F.: Texture analysis of medical images. Clin. Radiol. 59(12), 1061-1069 (2004)

4. Hajek, M., Dezortova, M., Materka, A., Lerski R.A. (eds.): Texture Analysis for Magnetic Resonance Imaging. Med4Publishing, Prague, Czech Republic (2006)

5. Nailon, W.H.: Texture Analysis Methods for Medical Image Characterisation. In: Mao, Y. (ed.) Biomedical Imaging, pp. 75-100. InTech Open (2010)

6. Nguyen, F., Cherel, Y., Guigand, L., Goubault-Leroux, I., Wyers, M.: Muscle lesions associated with dystrophin deficiency in neonatal golden retriever puppies. J. Comp. Pathol. 126(2-3), 100-108 (2002)

7. Valentine, B.A., Cooper, B.J., Cummings, J.F., de Lahunta, A.: Canine X-linked muscular dystrophy: morphologic lesions. J. Neurol. Sci. 7(1), 1-23 (1990)

8. Nguyen, F., Guigand, L., Goubault-Leroux, I., Wyers, M., Cherel, Y.: Microvessel density in muscles of dogs with golden retriever muscular dystrophy. Neuromuscul. Disord. 15(2), 154-163 (2005)

9. De Certaines, J.D., Larcher, T., Duda, D., Azzabou, N., Eliat, P.A., et al.: Application of texture analysis to muscle MRI: 1-What kind of information should be expected from texture analysis? EPJ Nonlinear Biomed. Phys. 3(3), 1-14 (2015)

10. Lerski, R.A, de Certaines, J.D., Duda, D., Klonowski, W., Yang, G., et al.: Application of texture analysis to muscle MRI: 2 technical recommendations. EPJ Nonlinear Biomed. Phys. 3(2), 1-20 (2015)

11. Herlidou, S., Rolland, Y., Bansard, J.Y., Le Rumeur, E., de Certaines, J.D.: Comparison of automated and visual texture analysis in MRI: characterization of normal and diseased skeletal muscle. Magn. Reson. Imaging 17(9), 1393-1397 (1999)

12. Skoch, A., Jirak, D., Vyhnanovska, P., Dezortova, M., Fendrych, P., et al.: Classification of calf muscle MR images by texture analysis. Magn. Reson. Mater. Phy. 16(6), 259-267 (2004)

13. Fan, Z., Wang, J., Ahn, M., Shiloh-Malawsky, Y., Chahin, N., et al.: Characteristics of magnetic resonance imaging biomarkers in a natural history study of golden retriever muscular dystrophy. Neuromuscul. Disord. 24(2), 178-191 (2014) 
14. Sikio, M., Harrison, L.C., Nikander, R., Ryymin, P., Dastidar, P., et al.: Influence of exercise loading on magnetic resonance image texture of thigh soft tissues. Clin. Physiol. Funct. Imaging 34(5), 370-376 (2014)

15. Nketiah, G., Savio, S., Dastidar, P., Nikander, R., Eskola, H., Sievanen, H.: Detection of exercise load-associated differences in hip muscles by texture analysis. Scand. J. Med. Sci. Sports 25(3), 428-434 (2015)

16. Duda, D.: Medical image classification based on texture analysis. PhD Thesis, University of Rennes 1, Rennes, France (2009)

17. Thibaud, J.L., Azzabou, N., Barthelemy, I., Fleury, S., Cabrol, L., et al.: Comprehensive longitudinal characterization of canine muscular dystrophy by serial NMR imaging of GRMD dogs. Neuromuscul. Disord. 22, Suppl. 2, S85-S99 (2012)

18. Gonzalez, R.C., Woods, R.E.: Digital Image Processing, 2nd ed. Addison-Wesley, Reading, MA, USA (2002)

19. Lerski, R., Straughan, K. Shad, L., Boyce, D., Bluml, S., Zuna, I.: MR image texture analysis - an approach to tissue characterization. Magn. Reson. Imaging 11(6), 873-887 (1993)

20. Haralick, R.M., Shanmugam, K., Dinstein, I.: Textural features for image classification. IEEE Trans. Syst., Man, Cybern., Syst. SMC-3(6), 610-621 (1973)

21. Galloway, M.M.: Texture analysis using gray level run lengths. Comp. Graph. and Im. Proc. 4(2), 172-179 (1975)

22. Chu, A., Sehgal, C.M., Greenleaf, J.F.: Use of gray value distribution of run lengths for texture analysis. Pattern Recogn. Lett. 11(6), 415-419 (1990)

23. Albregtsen, F., Nielsen, B., Danielsen, H.E.: Adaptive gray level run length features from class distance matrices. In: Sanfeliu, A., Villanueva, J.J., Vanrell, M., Alqukzar, R., Crowley, J., Shirai, Y. (eds.) 15th International Conf. on Pattern Recogn. 2000, vol. 3, pp. 738-741, IEEE Press, Los Alamitos, CA, USA, (2000)

24. Weszka, J.S., Dyer, C.R., Rosenfeld, A.: A comparative study of texture measures for terrain classification. IEEE Trans. Syst., Man Cybern. 6(4), 269-285 (1976)

25. Chen, E.L., Chung, P.C., Chen, C.L., Tsai, H.M., Chang, C.I.: An automatic diagnostic system for CT liver image classification. IEEE Trans. Biomed. Eng. 45(6), 783-794 (1998)

26. Laws, K.I.: Textured image segmentation. PhD Thesis, University of Southern California, Los Angeles, CA, USA (1980)

27. Hall, M., Frank, E., Holmes, G., Pfahringer, B., Reutemann, P., Witten, I.H.: The WEKA data mining software: an update. SIGKDD Explorations 11(1), 10-18 (2009)

28. Vapnik, V.N.: The Nature of Statistical Learning Theory, 2nd ed. Springer, New York, NY, USA (2000).

29. Platt, J.C.: Fast Training of Support Vector Machines Using Sequential Minimal Optimization. In: Scholkopf, B., Burges, C.J.C., Smola, A.J. (eds.) Advances in Kernel Methods - Support Vector Learning, pp. 185-208, MIT Press, Cambridge, MA, USA (1998)

30. Rojas, R.: Neural Networks. A Systematic Introduction. Springer-Verlag, Berlin, Germany (1996)

31. Hosmer, D.W., Lemeshow, S., Sturdivant, R.X.: Applied Logistic Regression, 3rd ed. John Wiley \& Sons, Inc., Hoboken, NJ, USA (2013)

32. Breiman, L.: Random Forests. Mach. Lear. 45(1), 5-32 (2001)

33. Freund, Y., Shapire, R.: A decision-theoretic generalization of online learning and an application to boosting. J. Comput. System Sci. 55(1), 119-139 (1997)

34. Quinlan, J.: C4.5: Programs for Machine Learning. Morgan Kaufmann, San Francisco, CA, USA (1993) 